\title{
Destrucción del patrimonio, construcción del no lugar, Huasca de Ocampo, Hidalgo.
}

\author{
Mayela Benavides a, *, Carlos Alfredo Bigurra-Alzati a , Francisco Omar Lagarda-García a \\ a Profesor Investigador del Área Académica de Ingeniería y Arquitectura de la Universidad Autónoma del Estado de Hidalgo, integrante del Grupo de Investigación \\ "Arquitectura, Tecnología y Habitabilidad"
}

\section{Resumen}

Con el propósito de iniciar o incrementar la actividad turística en ciertas localidades, se busca capitalizar el patrimonio construido, no construido, tangible e intangible de estas, las cuales son intervenidas bajo diversos criterios. Tales acciones son efectuadas o promovidas por los niveles de gobierno, federal, estatal y municipal de forma conjunta o individual. En ocasiones resultan favorables, en otras, contraproducentes porque no contribuyen a la preservación del patrimonio y favorecen la construcción de un 'no lugar'. Con el propósito de estudiar este problema se utilizó un método cualitativo basado en entrevistas a los actores sociales locales para estudiar las tres variables propuestas. Entre los hallazgos destaca que no todas las intervenciones efectuadas para contribuir a contar con un pueblo con un mayor atractivo turístico tuvieron las repercusiones contempladas. Como fue la terraza edificada sobre el río, la cual está abandonada y en franco deterioro. El propósito de esta fue utilizarla para reinstalar a los prestadores de servicios turísticos, quienes actualmente están ubicados enfrente de la iglesia y el jardín central. Situación que coloca en riesgo la permanencia del pueblo en el Programa Pueblos Mágicos (PPM), que establece que en el espacio público no debe haber vendedores ambulantes.

Palabras Clave: patrimonio, destrucción/construcción, 'no lugar', pueblos mágicos, Huasca, Hidalgo.

\section{Introducción}

El PPM fue instituido en el país hace diecisiete años, actualmente pertenecen a él 111 pueblos, los cuales fueron elegidos porque se consideró que cuentan con un patrimonio que contribuye a la captación de turismo. Las intervenciones que les son realizadas conllevan impactos, en el presente trabajo se estudió una de las problemáticas de Huasca de Ocampo, Hidalgo, como son: Las intervenciones efectuadas o promovidas por los gobiernos o los propietarios de las edificaciones con el propósito de que el pueblo resulte atractivo turísticamente, a veces resultan favorables, en otras ocasiones son contraproducentes porque no contribuyen a la preservación del patrimonio de un lugar, a veces más bien a la destrucción, resultando en la construcción de un 'no lugar'. La pregunta central del trabajo fue ¿De qué forma se destruye o se preserva el patrimonio de un lugar que se busca despunte como destino turístico? El objetivo fue: Estudiar las formas de construcción o destrucción del patrimonio del primer Pueblo Mágico del país que fue integrado al PPM.

La metodología utilizada para cumplir con el objetivo planteado consistió en efectuar una revisión bibliográfica de aspectos como son los teórico metodológicos conceptuales basados en los documentos de ICOMOS y del PPM, así como información sobre el patrimonio del pueblo y la política pública que incide directamente en este. Con el propósito de analizar los resultados de las intervenciones al patrimonio implementadas en Huasca, se estudiaron desde la perspectiva de diversos actores locales. Para lo cual se utilizó un método cualitativo, entrevistas basadas en un guion de historias de vida, realizadas en la localidad en los años 2016 y 2017. De entre ellos se eligieron a comerciantes, guías turísticos, residentes, presidente del Comité del Pueblo Mágico y coordinadores de turismo municipal. Las variables estudiadas fueron territorio, patrimonio y política pública. El corpus obtenido fue analizado por medio de un programa de análisis cualitativo de datos (Atlas Ti).

\section{Materiales y métodos}

Días antes de que concluyera el año 2017, el Secretario de Turismo a nivel federal publicó las Reglas de Operación del Programa de Desarrollo Regional Turístico Sustentable y Pueblos Mágicos vigentes en el Ejercicio Fiscal 2018, estas inician con una recapitulación de las metas nacionales del Plan Nacional de Desarrollo (PND) 2013-2018 que consiste en lograr un México Próspero, elevando la productividad del país, incrementando el crecimiento potencial de la economía y el bienestar de las familias. El objetivo

4.11 del PND es aprovechar el potencial turístico del país y con ello generar una mayor derrama económica para este. En lo referente al Programa Sectorial de Turismo, en el objetivo 2 , se establece “...la necesidad de promover un turismo sustentable y de calidad que ofrezca productos y servicios innovadores, con mayor valor agregado y con una adecuada articulación de la cadena de valor, en aras de fortalecer la competitividad del turismo mexicano y que haga que la industria turística se desarrolle en beneficio de los mexicanos."

* Autor en correspondencia.

Correos electrónicos: mayela.benavides@gmail.com (Mayela Benavides) 
(SEGOB 2017). Con el propósito de contribuir a la consolidación de destinos y el fomento de los productos turísticos se busca mejorar la infraestructura y equipamiento en las regiones turísticas, por medio del Programa Presupuestario S248 "Programa de Desarrollo Regional Turístico Sustentable y Pueblos Mágicos (PRODERMAGICO)".

El PPM fue instituido en el 2001 por la Secretaría de Turismo Federal como una política turística, con la finalidad de crear una marca distintiva del turismo de México, y así contribuir a los objetivos de desarrollo por medio de que este sea una actividad que eleve los niveles de bienestar, mantenga y acreciente el empleo, fomente y reditúe las inversiones en las localidades reconocidas por el PPM. Lo anterior fue asentado en el documento denominado Guía de incorporación y permanencia, Pueblos Mágicos, así como la definición del concepto Pueblo Mágico: "Una localidad que a través del tiempo y ante la modernidad, ha conservado, valorado y defendido, su herencia histórica, cultural y natural; y la manifiesta en diversas expresiones a través de su patrimonio tangible e intangible...que tiene atributos únicos, simbólicos, historias auténticas, hechos trascendentes, cotidianidad, que significa una gran oportunidad para el aprovechamiento turístico atendiendo a las motivaciones y necesidades de los viajeros." (SECTUR 2014). La finalidad de este programa es promover el desarrollo económico local basado en el patrimonio que ostentan las comunidades rurales del país.

El Consejo Internacional de Monumentos y Sitios, ICOMOS* (International Council on Monuments and Sites) es una asociación civil no gubernamental, cuya sede es París, Francia, está ligada a la ONU por medio de la $\mathrm{UNESCO}^{\dagger}$. Fundada en 1965, con la finalidad de dar seguimiento a la Carta de Venecia de 1964 que fue elaborada en el II Congreso Internacional de Arquitectos y Técnicos de Monumentos Históricos. Entre las funciones del consejo está proponer los sitios que son evaluados para ser declarados Patrimonio Cultural de la Humanidad. También busca la utilización de teorías, metodologías y tecnologías en la conservación, protección y valorización de monumentos y sitios con valor cultural.

Desde hace más de cincuenta años existe ICOMOS Mexicano AC, organismo que realiza aportaciones a la agenda del patrimonio mundial. A lo que se añade que por decreto presidencial de forma permanente es consultor especializado para el gobierno mexicano ${ }^{\ddagger}$. México ha participado con ICOMOS desde su creación y es uno de los cuatro países que se suscribieron a la Carta de Venecia.

\footnotetext{
* ICOMOS es una organización internacional no gubernamental que trabaja para la conservación de monumentos y sitios en todo el mundo. https://www.icomos.org/fr/ consultada el 29.05.18

$\dagger$ Para la UNESCO, el patrimonio cultural inmaterial es el conjunto de creaciones basadas en la tradición de una

comunidad cultural expresada por un grupo o por individuos y que reconocidamente responden a las expectativas de una comunidad en la medida en que reflejan su identidad cultural y social.

* ICOMOS Mexicano lo realiza a través de relaciones interinstitucionales tanto con el gobierno federal como con los estatales y municipales, con la academia y la investigación, con organismos de la sociedad civil, asociaciones, colegios y representantes de profesionales, fundaciones varias, fideicomisos así como con el H. Congreso de la Unión y los congresos locales, cámaras, grupos empresariales. Así como también, con empresas nacionales y globales. No se encontró información sobre que el organismo asesore a SECTUR sobre la preservación y conservación del patrimonio nacional
}

La Carta de Venecia es un referente importante en la conservación del patrimonio porque confiere a la humanidad la capacidad de valorar su patrimonio y la responsabilidad de salvaguardarlo. Aunado a ello están las definiciones donde destaca que el monumento histórico no solo es la obra arquitectónica, sino también el conjunto rural y urbano, porque ellos son quienes proporcionan el panorama completo de la civilización, por modestos que puedan ser. Conservación y preservación incluye la obra de arte y el testimonio histórico por medio de la totalidad de las ciencias y las técnicas.

En el año de 1999, ICOMOS publicó dos documentos, la Carta del Patrimonio Vernáculo Construido y La Gestión del Turismo en los Sitios con Patrimonio Significativo. En la primera destaca la importancia que este tipo de herencia, la cual es grandemente apreciada por los pueblos, por estar ligado a su vida cotidiana y poseer la capacidad de mostrar la historia y actualidad de esta. Por la dignidad de la humanidad se debiera conservar su armonía porque muestra la relación de la comunidad con el territorio. Además alerta que este patrimonio está en riesgo por la homogenización cultural y arquitectura, razón por la que los gobiernos y especialistas deben cuidarlo. La segunda precisa que el patrimonio natural y cultural pertenece a todos los pueblos, lo que debiera derivar en el derecho y responsabilidad de comprenderlo, valorarlo y conservarlo.

El concepto de patrimonio es amplio e incluye sus entornos tanto naturales como culturales, estos son los paisajes, los sitios históricos, los emplazamientos y entornos construidos, así como la biodiversidad, los grupos de objetos diversos, las tradiciones pasadas y presentes, y los conocimientos y experiencias vitales. Registra y expresa largos procesos de evolución histórica, constituyendo la esencia de muy diversas identidades nacionales, regionales, locales, indígenas y es parte integrante de la vida moderna. Es un punto de referencia dinámico y un instrumento positivo de crecimiento $\mathrm{e}$ intercambio. La memoria colectiva y el patrimonio cultural distintivo de cada comunidad o localidad son insustituible y fundamento primordial para el desarrollo no solo actual sino futuro.

En estos tiempos de creciente globalización, la protección, conservación, interpretación y presentación de la diversidad cultural y del patrimonio cultural de cualquier sitio o región es un importante desafío para cualquier pueblo en cualquier lugar del mundo. Sin embargo, lo normal debiera ser que cada comunidad o grupo se responsabilice de la conservación y gestión de su patrimonio, teniendo en cuenta las normas internacionalmente reconocidas $y$ aplicadas de forma adecuada.

Un objetivo fundamental de la gestión del patrimonio consiste en comunicar su significado y la necesidad de su conservación tanto a la comunidad anfitriona como a los visitantes. El acceso físico, intelectual y/o emotivo, sensato y bien gestionado a los bienes del patrimonio, así como el acceso al desarrollo cultural, constituyen al mismo tiempo un derecho y un privilegio.

Esto conlleva la responsabilidad de respetar los valores del patrimonio natural o cultural, así como los intereses y patrimonios de la sociedad receptora de los pueblos indígenas conservadores de su patrimonio o de los poseedores de propiedades históricas, así como la obligación de respetar los paisajes y las culturas a partir de las cuales se ha desarrollado el patrimonio. 
La conceptualización de ICOMOS sobre patrimonio está basada en que este posee cualidades que lo convierten en un lugar que permiten palpar los elementos distintivos de este como son los elementos edificados y tradiciones entre otros, además son documentos vivos de los procesos de evolución a través de la historia que contribuyen a su identidad. En oposición están los 'no lugares', a lo que Michael de Certeau (1996) le concede una cualidad negativa del lugar, por considerarlo una ausencia de lugar en sí mismo, definió el espacio como un lugar que es animado por el total de los movimientos y acciones que en él efectúan quienes lo habitan, conforma su existencia y lo convierten en un lugar practicado y lo transforman en espacio; es su acción y la práctica humana asociada lo que distingue su espacio de un lugar.

En un sentido similar, Augé (2004) emplea el término de lugar y no espacio para hablar del espacio simbolizado. Tanto el concepto de espacio de De Certeau como el de lugar de Augé coinciden en las características de un lugar antropológico, que es el lugar practicado, el lugar que es identificado y a la vez identifica a quienes lo habitan. Este es el que es considerado como patrimonio inmaterial que muestra la magia del lugar, y al que el PPM acude para su existencia y la promoción del turismo.

\section{Contexto del primer pueblo mágico de México, Huasca de Ocampo, Hidalgo}

El municipio de Huasca de Ocampo por sus atractivos y ubicación cuenta con distinciones como es el que su cabecera municipal, con el mismo nombre, desde el 2001, es el primer pueblo del PPM, a partir de mayo del 2017 junto con otros ocho municipios de Hidalgo, es parte del Geoparque Mundial de la UNESCO Comarca Minera, Hidalgo por el valor y belleza natural de 31 geositios que muestran la historia de la minería y la metalurgia.

La superficie del municipio es 302.760 kilómetros cuadrados, el $1.5 \%$ de la superficie del estado, con una altitud que varía entre los 1800 y 2800 MSNM, está a 34 kilómetros de Pachuca, capital de este. Colinda al norte con Veracruz; al sur con los municipios de Tulancingo y Omitlán de Juárez; al este con el de Acatlán y al oeste con los de Atotonilco el Grande y Omitlán de Juárez. Su orografía es consecuencia de que el $70 \%$ de su área pertenece al eje Neovolcánico, por lo que posee llanuras y una pequeña proporción de sierra. El 30\% del resto del municipio está en la sierra madre oriental, territorio formado casi totalmente por cañones. La influencia de la Revolución Industrial

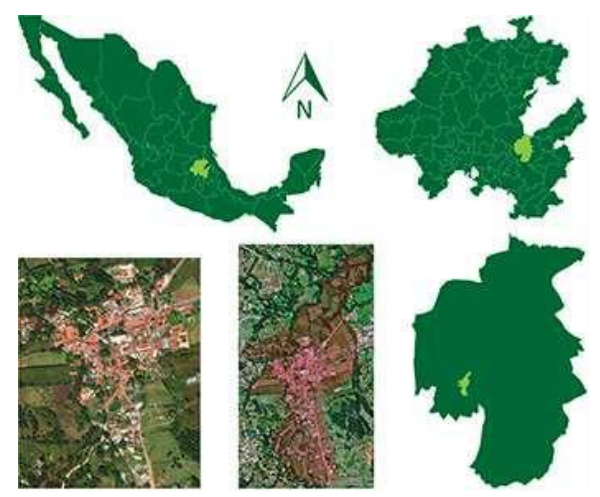

Figura 1: Localización de la cabecera municipal de Huasca de Ocampo y su respectiva imagen satelital (elaborada por Bigurra C. 2018).
Localización de la cabecera municipal de Huasca de Ocampo y su respectiva imagen satelital (elaborada por Bigurra C. 2018)

Su patrimonio construido está conformado principalmente por la Iglesia de San Juan Bautista, la Plaza Ocampo y el Monumento a la Independencia. Sus haciendas gozan de prestigio regional y funcionan como centros turísticos, destacando las de: San Miguel Regla, Santa María Regla y la de San Juan Bautista. En el municipio entre sus sitios de naturaleza y aventura están el Parque Ecoturístico Bosque de las Truchas en San Miguel Regla, los Prismas Basálticos en Santa María Regla, el Desarrollo Ecoturístico Bosques del Zembo, el Huariche en Ojo de Agua, el Arquito en Los Reyes Tepezala, la Barranca de Aguacatitla y el Centro Ecoturístico el Lago en San Miguel Regla. En los sitios ecoturísticos existen lagos, cascadas, bosques y cañadas, donde se puede también practicar turismo deportivo, en las haciendas mencionadas se realiza también turismo de negocios

El patrimonio cultural del municipio se puede apreciar en sus más de treinta festividades religiosas, de las que destaca la Semana Santa en Huasca, la del patrono del pueblo San Juan Bautista que se conmemora el 24 de junio. La gastronomía es parte del orgullo de los locales, la cual consta de truchas preparadas de alrededor de veinte formas distintas, barbacoa, escamoles (hueva de la hormiga), chinicuiles (gusanos de la raíz del maguey), chichas (gusanos de la penca del maguey), alverjones, caldo de hongos entre otros.

Con el propósito de dar a conocer el patrimonio del que se enorgullecen los originarios, los actores sociales involucrados en el desarrollo de la actividad turística diseñaron varios recorridos turísticos, los cuales tienen el propósito de mostrar su vasto patrimonio compuesto por elementos naturales, construidos, culturales y artesanales:

- Recorrido Huasca Tradicional: Santa María Regla, San Antonio Regla, Prismas Basálticos y Fábrica de Rompope.

- Recorrido Huasca al Natural: Peña del Aire, Santa María Regla, San Antonio Regla, Fábrica del Rompope.

- Recorrido Huasca Mágico: Museo de los Duendes, San Miguel Regla, Fábrica del Rompope y Centro Histórico de Huasca

\section{La política turística implementada en Huasca de Ocampo}

La administración municipal con el deseo de preservar el patrimonio de la cabecera municipal instituyó reglamentos y programas que contribuyan al mejoramiento de la imagen urbana de esta. Entre ellos está el Reglamento de anuncios e imagen urbana, Normatividad para preservar la unidad y coherencia arquitectónica de las edificaciones históricas, Programa de remozamiento de fachadas y borrar grafitis, Concientizar a la ciudadanía del cuidado y preservación de la imagen, Adecuación de paraderos de taxis, canal de contención pluvial y efectuar empedrados, Jornadas de limpieza semestrales, limpieza y recolección de basura diariamente y lavado bimestral de calles principales, Programa periódico para la presa de San Antonio para la eliminación el lirio acuático y recolección de basura, entre otros.

Antes de que concluyera el año 2002, la Presidencia Municipal de Huasca de Ocampo con el propósito de preservar la arquitectura tradicional del centro histórico de la cabecera municipal y este sea un legado para las futuras generaciones 
publicó un decreto que es el Reglamento para la Protección y Conservación del Centro Histórico: En este reconoce que para tal efecto es necesario que se efectúe el rescate y mantenimiento de este como de las iglesias y haciendas que conforman el patrimonio municipal. En el documento se estableció que el cumplimiento de este no se haga bajo una orientación negativa, sino más bien orientadora y preventiva.

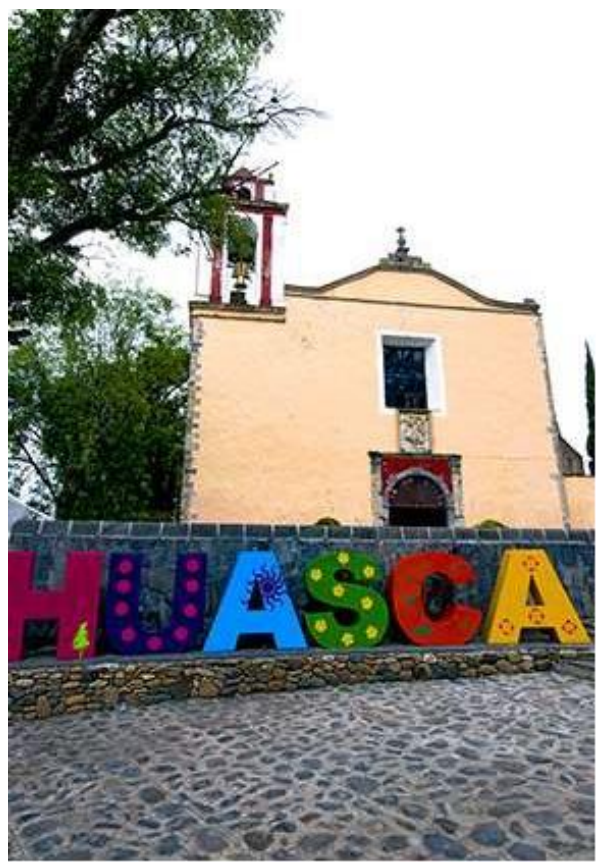

Figura 2: El centro de Huasca adornado con el nombre del Pueblo Mágico. Al fondo se aprecia la Iglesia de San Juan Bautista (Bigurra C. 2018).

La Agenda de Competitividad Turística de Huasca de Ocampo fue elaborada en el 2014 por diferentes instituciones gubernamentales y educativas por iniciativa de la Secretaría de Turismo, en ella están asentados más de una decena de análisis realizados con la herramienta de planeación estratégica FODA $^{*}$, es importante destacar el de la evaluación de Protección del paisaje y del territorio, natural y urbano. Para lo que esta determinó como problemática 'El crecimiento desordenado del pueblo y el deterioro de la imagen urbana que disminuye su atractivo y su competitividad como destino turístico' (SECTUR 2014). La recomendación que realizó para que el pueblo esté en la 'situación deseable' es que mejore el ordenamiento del territorio y con ello se logren algunos objetivos como son incrementar la percepción positiva que originarios y turistas realizan de ella, el consejo contribuiría a asegurar la conservación del patrimonio histórico, cultural, natural, así como para obtener una imagen urbana atractiva.

\section{Intervenciones al territorio que inciden en el patrimonio tangible, construido y natural}

La cabecera municipal, Huasca de Ocampo, con la finalidad de que fuera reconocida y permanezca siendo Pueblo Mágico, debe cumplir con las premisas requeridas por el PPM, por esta razón le han efectuado diferentes intervenciones,

\footnotetext{
* El análisis FODA son siglas que representan el estudio de las Fortalezas, Oportunidades, Debilidades y Amenazas, de una empresa un mercado, o sencillamente a una persona, este acróstico es aplicado a cualquier situación, en el cual, se necesite un análisis o estudio
}

algunas de ellas son el resultado de la planeación pública, otras por iniciativa de los propietarios de las edificaciones. Sobre las primeras se puede mencionar que tanto a la iglesia se Santa María Regla y los portales localizados enfrente de esta, a ambos les fueron eliminados el aplanado que tenían con el propósito de que la piedra quedara al descubierto. El atrio de la iglesia fue circundado con una barda. Conjuntamente al remozamiento del espacio central se talaron algunos árboles del jardín central, así como dos jacarandas que adornaban el frente de la iglesia.

Con la finalidad de contar con un mejor y mayor número de servicios hoteleros se restauró la Hacienda de San Miguel Regla, bajo la premisa de conservar las tipologías y materiales originales de las puertas, ventanas y vigas de techos, a lo que se añadió respetar la paleta de color original. En contraste con la iglesia y los portales, los muros de las haciendas no contaban con aplanados. A las construcciones históricas que se usan para el hospedaje se añadió la reutilización de edificaciones existentes, como la de nuevas con el objetivo de que sirvan de hoteles y exista un mayor número de habitaciones. El sector público añadió a la infraestructura turística centros y parque recreativos.

Algunos dueños de casas del pueblo ante la expectativa que conlleva el que este fuera promovido turísticamente desde el ámbito federal $y$ el deseo de contar con un mejor posicionamiento o una posibilidad de convertirse en prestadores de servicios turísticos, en algunos casos remodelaron o reconstruyeron sus casas, de un piso a dos niveles con diseño contemporáneo. Desafortunadamente otros derribaron su propiedad total o parcialmente y construyeron con nuevas tipologías. Ante lo sucedido el municipio intervino estableciendo el Reglamento para la Protección y Conservación del Centro Histórico con el fin de preservar el patrimonio construido y promoviendo que las edificaciones reciban mantenimiento de forma cíclica.

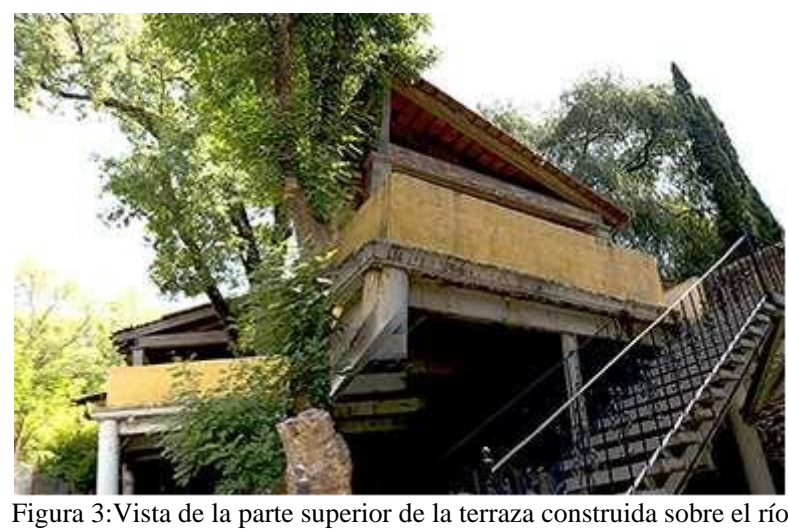
(Bigurra C. 2018).

Unos pocos años después de que la cabecera municipal fuera reconocida como Pueblo Mágico la Secretaría de Turismo del Gobierno del Estado con presupuesto del Gobierno Federal, construyó una terraza sobre el Río Huascazaloya-Hueyepan $^{\dagger}$ con un costo aproximado de 30 millones de pesos. La estrategia de la obra fue mejorar la

\footnotetext{
† El río desemboca en la Presa San Antonio, y se localiza a menos de cien metros de la calle principal del pueblo, la antigua carretera Hidalgo Tulancingo-Pachuca, así como del Quiosco de Huasca de Ocampo, de los arcos, de la iglesia, el jardín principal y la presidencia municipal
} 
imagen urbana del espacio público, del jardín frente a la iglesia y la explanada de los arcos por medio de reubicar el comercio ambulante de las áreas mencionadas en la nueva edificación.

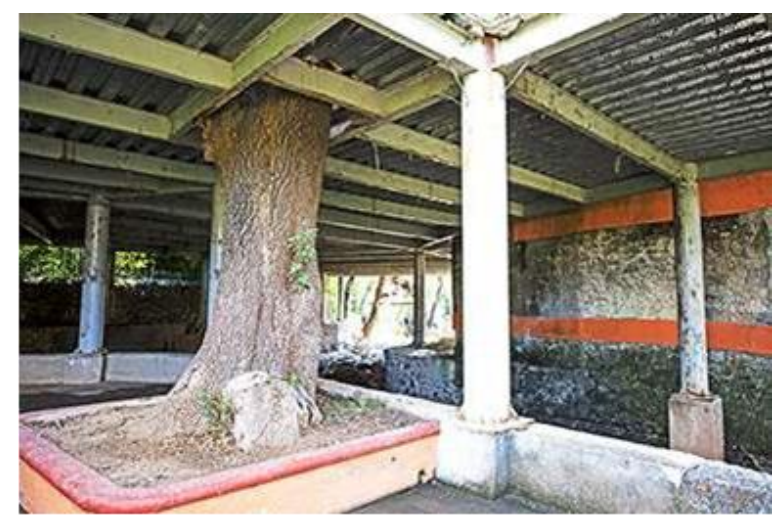

Figura 4: Vista inferior de la terraza, columnas y arriates, en el lecho del río (Bigurra C. 2016).

El concepto de diseñó de la terraza tuvo como fundamento la emulación de los arcos de piedra del túnel que conducen a la Hacienda de Santa María Regla. Fue construida con estructura metálica, el piso con tablones de madera y la techumbre con teja. Está en desuso y en deterioro porque los comerciantes nunca la utilizaron y no recibe mantenimiento alguno. Aparte de que la inversión no fue capitalizada, degradó el paisaje y patrimonio natural del lecho del río que conformó el imaginario de los locales, conformado por todo el escenario, incluyendo el sonido que produjo su caudal y la velocidad con la que bajaba de las montañas, hace algunos años este fue desviado por lo que los originarios recuerdan con añoranza. Con el tiempo se ha incrementado el número de comerciantes localizados en la vía pública, así como el aumento de tipos de mercancías que se ofertan.

\section{Acciones para promover el patrimonio cultural}

Los expertos en patrimonio inmaterial consideran que para salvaguardar este se debe preservar la magia. La de Huasca inicia con lo que significa su nombre en lengua Nahoa, lugar de regocijo, agua y pájaros, lo que los originarios traducen en que es un sitio donde se quiere estar. Coinciden con ello cuando dicen que es un lugar tranquilo, donde se vive relajado y respira aire fresco porque está rodeado de hermosas áreas naturales con manantiales y árboles, de las que pueden disfrutar acompañados de sus familias.

La difusión de la magia del pueblo ha sido efectuada a través de las novelas filmadas en él y programas de televisión sobre sus atractivos, así como por artículos publicados en diferentes medios de comunicación, revistas y periódicos.

Hace unos años, veinte jóvenes interesados en participar en el desarrollo de la actividad turística fueron capacitados por el Programa Vigías del Patrimonio Cultural del Consejo Nacional para la Cultura y las Artes (CONACULTA), que impartió un diplomado de un método de investigación para detectar y promover el patrimonio cultural local. En él los participantes diseñaron recorridos turísticos para difundir el patrimonio: gastronomía, danza, alfarería18. Este último incluye que los turistas visiten algunos talleres de cerámica y en uno de ellos elaboren una pieza del material, la experiencia sensorial que conlleva la actividad contribuye a la valoración de esta artesanía representativa del pueblo.

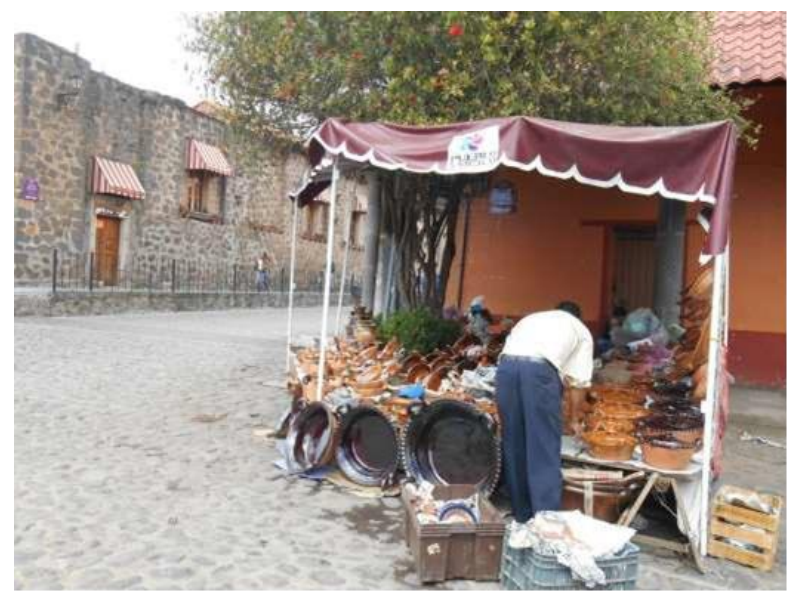

Figura 5: Calle principal del pueblo en la cual un artesano local exhibe su mercancía artesanal, el toldo del puesto ostenta el logotipo del PPM (Benavides M. 2016).

\section{Reflexión sobre patrimonio y no lugar de Huasca de Ocampo}

El PPM fue instrumentado teniendo como fundamento el PND, con la finalidad de contribuir al cumplimiento del objetivo planteado en este de un México prospero basado en la productividad'. Para ello alude al patrimonio que poseen los pequeños pueblos de México, el cual funciona como activo que debe capitalizarse en aras de desarrollar la actividad turística porque supone que ayudará a mejorar el nivel de bienestar de los residentes de las localidades involucradas.

Es primordial considerar el posicionamiento de ICOMOS en cuanto a la importancia de conservar y preservar el patrimonio vernáculo, porque este es esencial para la identidad de un pueblo tanto como espacio, como sociedad, la destrucción de los bienes incidirá negativamente en la percepción de los locales como del turismo. Es conveniente tener presente que la globalización está simbolizada en México por los programas turísticos como el PPM, en algunos pueblos pertenecientes a este ha contribuido a la unificación de las cualidades tangibles e intangibles de estos y con ello a la estructuración de los mencionados 'no lugares' por la pérdida de elementos vitales que contribuyen a la conformación de su patrimonio y con ello a su identidad.

En el caso del primer Pueblo Mágico del país, Huasca de Ocampo, 'El crecimiento desordenado del pueblo y el deterioro de la imagen urbana disminuye su atractivo y su competitividad como destino turístico' (SECTUR 2014). Lo anterior es en parte resultado de que se realizaron intervenciones a las edificaciones por sus propietarios sin acatar reglamentación para estas. Las acciones de los poseedores de las propiedades responden a la sinergia que conlleva un programa que tiene como discurso la generación de empleos y la prosperidad que experimentarán las comunidades involucradas, juntamente con sus habitantes. La problemática expuesta por el FODA es resultado de que cuando se incluye un pueblo en el PPM no se instituyen acciones precisas que incidirán en la valoración del patrimonio vernáculo, y con ello en la preservación y no destrucción del 'lugar' en contraste con la construcción del 'no lugar'. Asimismo no se contemplan programas de amortiguamiento 
de las problemáticas que desencadena la implementación del programa.

La edificación de la terraza es también consecuencia de la problemática manifestada en el FODA, que inició con una incomprensión de lo que implica la conservación y preservación del total del patrimonio, que si se efectúa cabalmente incide positivamente en la imagen urbana. Porque para efectuar una intervención urbana no es suficiente solo materializar cualquier propuesta que se tenga. Debe ser sometida rigurosamente a una valoración de cómo contribuye a la preservación del patrimonio en su totalidad. En este caso se debió haber inventariado el patrimonio natural como el inmaterial avalado en las costumbres y en las tradiciones como en la magia que le atribuyen los originarios al río. Desafortunadamente la terraza no contribuyó a capitalizar el presupuesto federal que pudo ser utilizado en obras que redituaran a la imagen urbana en lugar del deterioro de ella. Esto porque no solucionó la problemática que trae consigo el ambulantaje, más bien creció, a tal grado que el pueblo podría estar en riesgo de perder su incorporación al PPM. Además perjudicó el patrimonio natural localizado en el corazón de la localidad y que antes de la intervención contribuyó a la magia del pueblo, de la cual podrían disfrutar también los turistas, existió la posibilidad de que conformará su imaginario, como lo es él de los originarios.

Ante lo anterior la trascendencia de contar con programas fuertemente fundamentados y planteados que tengan como objetivo preservar el patrimonio por medio de salvaguardar la magia, por esta razón la importancia del Programa Vigías del Patrimonio Cultural, que reunió a los actores sociales claves que participan o tienen el deseo de promover actividades que sean atractivas al turismo. En el curso resultó fundamental que los participantes inventariaran y describieran los elementos que componen el patrimonio, y basado en estos fue que realizaron las propuestas. A lo que se debiera añadir enumerar de qué forma se debe proteger para no atentar contra este. Resulta altamente conveniente contemplar acciones específicas para conservar, sino se destruye el patrimonio y se construye un 'no lugar'.

\section{English Summary}

\section{Destruction of the patrimony, construction of the no place, Huasca de Ocampo, Hidalgo}

\footnotetext{
Abstract

In order to initiate or increase tourist activity in certain localities, actions are taken to capitalize built, unbuilt, tangible and intangible heritage areas. These interventions are often low criteria and are carried out or promoted by the government at the federal, state and municipal levels, jointly or individually. Sometimes these interventions are favorable but other times they are counterproductive because they do not contribute to the preservation of heritage and lead to the creation of a 'no place.' In order to study this problem, a qualitative method based on interviews with local social actors was used to analyze three proposed variables. Among the findings are that not all the interventions made to contribute to a town contemplated all the possible repercussions. Such is the
}

case of the terrace built on the river, which is abandoned and deteriorating. The purpose of the terrace was to move the tourism service providers to this area. The service providers are currently in front of the church and the central garden which endangers the permanence of the town in the Magic Towns Program (PPM), given that this program establishes that in the public space areas there should not be street vendors.

Keywords:

Heritage, Destruction / construction, 'no place', Magical Towns, Huasca, Hidalgo

\section{Referencias}

Aguilar Criado, Encarnación (2005) Patrimonio y globalización: el recurso de la cultura en las Políticas de Desarrollo Europeas Cuadernos de Antropología Social, núm. 21, pp. 51-69 Universidad de Buenos Aires Buenos Aires, Argentina

Augé, Marc (2000) Los 'no lugares' espacios del anonimato. Una antropología de la sobremodernidad.

Tr. Margarita Mizraji, Editorial Gedisa S. A., Barcelona.

De Certeau, Michel (1996) La invención de lo cotidiano, México, Universidad Iberoamericana. INAFED (2010) Enciclopedia de los Municipios y Delegaciones de México, Estado de Hidalgo. INEGI (2015) Anuario Estadístico del Estado de Hidalgo 2015.

Gobierno del Estado de Hidalgo (2011) Plan de Desarrollo 2011-2016 del Estado de Hidalgo.

ICOMOS (1999) Carta Internacional sobre Turismo Cultural (La gestión del turismo en los sitios con patrimonio significativo)

http://www.planmaestro.ohc.cu/recursos/papel/cartas/1999-turismo.pdf consultada el 17.05.18

López-Levi, Liliana (2015). Pueblos mágicos mexicanos: magia, hechizo e ilusión. URBS. Revista de Estudios Urbanos y Ciencias Sociales, 5(2), 1326.

http://www2.ual.es/urbs/index.php/urbs/article/ view/llevi

Presidencia Municipal de Huasca de Ocampo, Hidalgo (2002) Reglamento para la Protección y Conservación del Centro Histórico http://huasca.hidalgo.gob.mx/transparencia/FRACCI\%C3\%93N\%20I/MA RCO\%20NORMATIV O/CENTRO\%20HISTORICO.pdf consultado el 19.06.18

Proyecto Geoparque Comarca Minera http://www.geoparquehidalgo.com/ consultada el 05.06.18

Secretaría de Gobernación, Diario Oficial de la Federación (2018) Las Reglas de Operación del Programa de Desarrollo Regional Turístico Sustentable y Pueblos Mágicos vigentes en el Ejercicio Fiscal https://www.google.com.mx/search?rlz=1C1CHBD_esMX792MX792\&q $=$ reglas $\% 20 \mathrm{de} \% 20$ operacion $\% 20$ pueblos $\% 20$ magicos $\%$ $202018 \&$ ved $=0$ ahUKEwj_-

622jLHbAhUEbq0KHYQjDPwQsKwBCC8oADAA\&biw=1280\&bih=64 5 consultado el 31.05.1

Secretaria de Turismo (2014) Agenda de Competitividad Turística del Destino Huasca de Ocampo, Pueblo Mágico

Secretaria de Turismo (2014) Guía de incorporación y permanencia Pueblos Mágicos

http://www.sectur.gob.mx/wp-content/uploads/2014/10/GUIA-FINAL.pdf consultada el 17.05.18

UNESCO (2003) Convención para la Salvaguardia del Patrimonio Cultural Inmaterial

http://www.planmaestro.ohc.cu/recursos/papel/cartas/2003-inmaterial.pdf consultada el 17.05.18

Valverde, Carmen, Liliana López Leví, Ana María Fernández (2015) Huasca de Ocampo, Hidalgo. Donde la magia inicia (coords.) López, Liliana, Valverde, Carmen, Ana María Fernández, María Elena Figueroa, Pueblos Mágicos. Una visión interdisciplinaria. Volumen I. México, UAM, UNAM. Pp 23-44. 